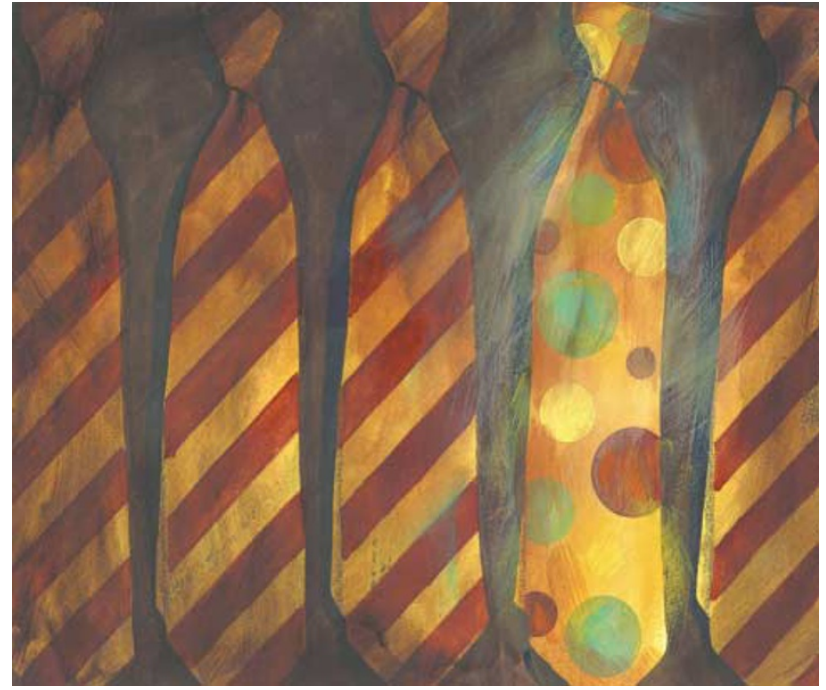

ION CHANNELS

\section{How the channel lost} its symmetry

Cyclic nucleotide-gated channels (CNGs) are crucial for signal transduction in the visual and olfactory systems. Similar to other channels that have six transmembrane domains, CNGs are tetrameric. In the case of rod photoreceptor CNGs, the tetramers are heteromeric complexes of the $\mathrm{A} 1$ and $\mathrm{B} 1 \mathrm{CNG}$ subunits. But how many subunits of each type are there in a given channel? Although indirect evidence indicated that rod CNG channels might have two $\mathrm{A} 1$ and two $\mathrm{B} 1$ monomers, two recent papers reach the surprising conclusion that they contain three $\mathrm{A} 1$ and one $\mathrm{B} 1$ subunits.

In the first paper, Weitz et al. used chemical crosslinking to measure the stoichiometry of native channels. As A1 and B1 subunits have different molecular weights, the authors predicted the existence of complexes of different mass, which depended on the number of $\mathrm{A} 1$ and $\mathrm{B} 1$ monomers that are present in the channel. In the second report, Zheng et al. established the stoichiometry of recombinant channels by using fluorescence resonance energy transfer between tagged subunits. This method depends on the ability of a stimulated fluorophore to transfer energy to a second fluorophore located within a short radius, and provides an excellent estimate of intra- and intermolecular distance.

Both studies provided evidence that rod CNG channels have three $\mathrm{A} 1$ and one B1 monomers. As B1 subunits confer rod CNG channels with specific functional properties, such as their modulation by $\mathrm{Ca}^{2+} / \mathrm{calmodulin}$, it will be important to discover how a single subunit fulfils this role. More importantly, the results pose questions about how channel assembly takes place and about the mechanisms that prevent two B1 subunits from integrating into a tetramer. The data also raise the intriguing possibility that other tetrameric channels have similar assembly restrictions. Last, as olfactory CNG channels consist of three different subunits (A2, A4 and B1b), the approaches of both groups might help unravel their stoichiometry.

Juan Carlos López

69) References and links

ORIGINAL RESEARCH PAPERS Weitz, D. et al. Subunit stoichiometry of the CNG channel of rod photoreceptors. Neuron 36, 881-889 (2002) | Zheng, J. et al. Rod cyclic nucleotidegated channels have a stoichiometry of three CNGA1 subunits and one CNGB1 subunit. Neuron 36, 891-896 (2002)

FURTHER READING Flynn, G. E. et al. Cyclic nucleotide-gated channels: shedding light on the opening of a channel pore. Nature Rev. Neurosci. 2, 643-651 (2001)

\title{
FUNCTIONAL NEUROIMAGING
}

\section{Visual learning visualized}

Our understanding of the neural processes of perceptual learning has benefited from both psychophysical studies in humans and electrophysiology in monkeys. Functional imaging has the potential to bridge the gap between these two approaches, as illustrated by Schwartz and colleagues. Their study provides evidence that learning a texture discrimination task produces local changes in primary visual cortex (V1), but not in higher visual cortical areas.

Subjects had to keep their vision centred on a screen and identify the orientation of a textured patch that appeared at one point in the visual field, using only one eye. Performance on this task improves with practice, but the improvement is specific to the trained eye and the retinal location at which the test patch appears. This suggests that the neural changes that mediate the improvement might be local to early visual areas, where neurons respond specifically to input from one eye and have relatively small receptive fields. Further evidence for local changes comes from recordings of neurons in monkey $\mathrm{V} 1$, which show that training on a similar task produces changes in the responses of neurons that respond to the trained area of retina and to the orientation of stimulus used in training.

However, feedback from higher areas of visual cortex could also be involved in perceptual learning. In particular, such learning in humans depends on attention, and higher areas such as V4 have been implicated in monkeys. Schwartz et al. used

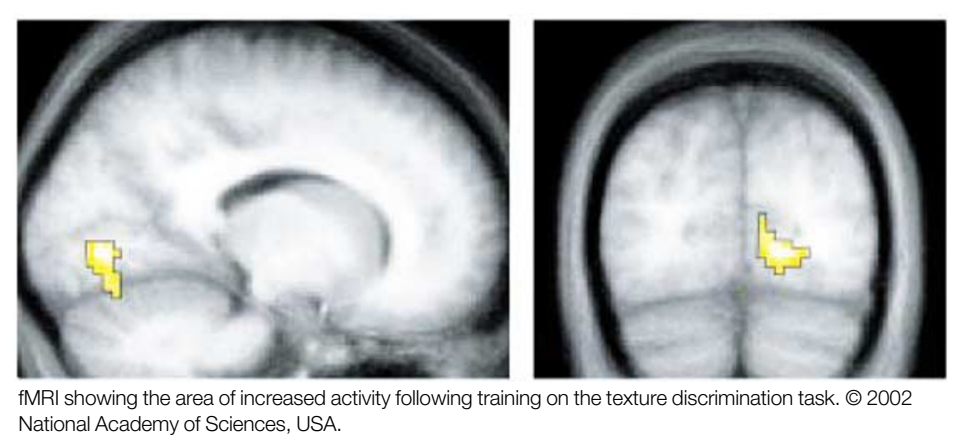

functional magnetic resonance imaging (fMRI) to look for changes in activity that resulted from intensive training on the texture discrimination task. They found that, one day after training, there was an increase in activity in just that area of V1 that responded to stimulation at the trained retinal location. This increase in activity emerged when the authors compared performance of the task with the trained eye against performance with the untrained eye. Other areas of cortex showed no change in activity.

The authors used an analysis of functional connectivity to look for changes in the amount of coupling between activity in different brain areas as a function of training. Significant functional coupling between remote brain areas and V1 during the untrained condition indicated that attentional control might be required at this initial stage. By contrast, performance of the task with the trained eye produced no increase in coupling, supporting the idea that perceptual learning of texture discrimination is mediated by plastic changes that are limited to the earliest levels of visual processing.

\section{Rachel Jones}

(2) References and links ORIGINAL RESEARCH PAPER Schwartz, S. et al. Neural correlates of perceptual learning: A functional MRI study of visual texture discrimination. Proc. Natt Acad. Sci. USA 21 November 2002 (doi:10.1073/pnas.242414599) FURTHER READING Gilbert, C. D. et al. The neural basis of perceptual learning. Neuron $\mathbf{3 1}$, 681-697 (2001)

WEB SITES

Encyclopedia of Life Sciences:

http://www.els.net/

Brain imaging: localization of brain functions 